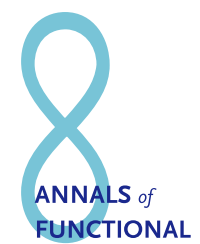

ANALYSIS
Ann. Funct. Anal. 7 (2016), no. 3, 421-433

http://dx.doi.org/10.1215/20088752-3605447

ISSN: 2008-8752 (electronic)

http://projecteuclid.org/afa

\title{
SHARP WEAK ESTIMATES FOR HARDY-TYPE OPERATORS
}

\author{
GUILIAN GAO, XIAOMIN HU, ${ }^{*}$ and CHUNJIE ZHANG \\ Communicated by J. Soria
}

\begin{abstract}
In this article, we prove the weak bound for an $n$-dimensional Hardy operator on a central Morrey space. Meanwhile, we obtain the precise operator norm, and we give the weak bounds for the conjugate Hardy operator on Lebesgue space with power weights. The corresponding operator norms are also computed. As an application, we obtain an estimate for the gamma function.
\end{abstract}

\section{INTRODUCTION}

Let $h$ and $h^{*}$ be the Hardy averaging operator and its conjugate Hardy operator,

$$
h f(x)=\frac{1}{x} \int_{0}^{x} f(t) d t, \quad h^{*} f(x)=\int_{x}^{\infty} \frac{f(t)}{t} d t, \quad x>0,
$$

respectively. The classical Hardy inequalities are

$$
\|h f\|_{L^{p}\left(\mathbb{R}^{+}\right)} \leq \frac{p}{p-1}\|f\|_{L^{p}\left(\mathbb{R}^{+}\right)}, \quad 1<p \leq \infty
$$

and

$$
\left\|h^{*} f\right\|_{L^{p}\left(\mathbb{R}^{+}\right)} \leq p\|f\|_{L^{p}\left(\mathbb{R}^{+}\right)}, \quad 1 \leq p<\infty .
$$

In addition, Hardy proved that the constants $p$ and $\frac{p}{p-1}$ above are the best possible; hence,

$$
\|h\|_{L^{p}\left(\mathbb{R}^{+}\right) \rightarrow L^{p}\left(\mathbb{R}^{+}\right)}=\frac{p}{p-1}, \quad 1<p \leq \infty
$$

Copyright 2016 by the Tusi Mathematical Research Group.

Received Aug. 4, 2015; Accepted Dec. 28, 2015.

${ }^{*}$ Corresponding author.

2010 Mathematics Subject Classification. Primary 42B35; Secondary 26D10, 46E30, 26 D15.

Keywords. Hardy operator, the conjugate Hardy operator, central Morrey space, weak central Morrey space, duality. 


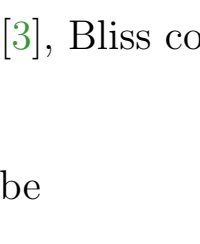

ANALYSIS
Ann. Funct. Anal. 7 (2016), no. 3, 421-433

http://dx.doi.org/10.1215/20088752-3605447

ISSN: 2008-8752 (electronic)

http://projecteuclid.org/afa

\title{
SHARP WEAK ESTIMATES FOR HARDY-TYPE OPERATORS
}

\author{
GUILIAN GAO, XIAOMIN HU, ${ }^{*}$ and CHUNJIE ZHANG \\ Communicated by J. Soria
}

\begin{abstract}
In this article, we prove the weak bound for an $n$-dimensional Hardy operator on a central Morrey space. Meanwhile, we obtain the precise operator norm, and we give the weak bounds for the conjugate Hardy operator on Lebesgue space with power weights. The corresponding operator norms are also computed. As an application, we obtain an estimate for the gamma function.
\end{abstract}

\section{INTRODUCTION}

Let $h$ and $h^{*}$ be the Hardy averaging operator and its conjugate Hardy operator,

$$
h f(x)=\frac{1}{x} \int_{0}^{x} f(t) d t, \quad h^{*} f(x)=\int_{x}^{\infty} \frac{f(t)}{t} d t, \quad x>0,
$$

respectively. The classical Hardy inequalities are

$$
\|h f\|_{L^{p}\left(\mathbb{R}^{+}\right)} \leq \frac{p}{p-1}\|f\|_{L^{p}\left(\mathbb{R}^{+}\right)}, \quad 1<p \leq \infty
$$

and

$$
\left\|h^{*} f\right\|_{L^{p}\left(\mathbb{R}^{+}\right)} \leq p\|f\|_{L^{p}\left(\mathbb{R}^{+}\right)}, \quad 1 \leq p<\infty .
$$

In addition, Hardy proved that the constants $p$ and $\frac{p}{p-1}$ above are the best possible; hence,

$$
\|h\|_{L^{p}\left(\mathbb{R}^{+}\right) \rightarrow L^{p}\left(\mathbb{R}^{+}\right)}=\frac{p}{p-1}, \quad 1<p \leq \infty
$$

Copyright 2016 by the Tusi Mathematical Research Group.

Received Aug. 4, 2015; Accepted Dec. 28, 2015.

${ }^{*}$ Corresponding author.

2010 Mathematics Subject Classification. Primary 42B35; Secondary 26D10, 46E30, 26 D15.

Keywords. Hardy operator, the conjugate Hardy operator, central Morrey space, weak central Morrey space, duality. 


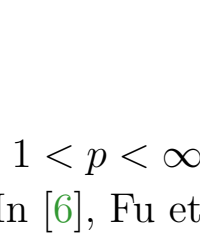

ANALYSIS
Ann. Funct. Anal. 7 (2016), no. 3, 421-433

http://dx.doi.org/10.1215/20088752-3605447

ISSN: 2008-8752 (electronic)

http://projecteuclid.org/afa

\title{
SHARP WEAK ESTIMATES FOR HARDY-TYPE OPERATORS
}

\author{
GUILIAN GAO, XIAOMIN HU, ${ }^{*}$ and CHUNJIE ZHANG \\ Communicated by J. Soria
}

\begin{abstract}
In this article, we prove the weak bound for an $n$-dimensional Hardy operator on a central Morrey space. Meanwhile, we obtain the precise operator norm, and we give the weak bounds for the conjugate Hardy operator on Lebesgue space with power weights. The corresponding operator norms are also computed. As an application, we obtain an estimate for the gamma function.
\end{abstract}

\section{INTRODUCTION}

Let $h$ and $h^{*}$ be the Hardy averaging operator and its conjugate Hardy operator,

$$
h f(x)=\frac{1}{x} \int_{0}^{x} f(t) d t, \quad h^{*} f(x)=\int_{x}^{\infty} \frac{f(t)}{t} d t, \quad x>0,
$$

respectively. The classical Hardy inequalities are

$$
\|h f\|_{L^{p}\left(\mathbb{R}^{+}\right)} \leq \frac{p}{p-1}\|f\|_{L^{p}\left(\mathbb{R}^{+}\right)}, \quad 1<p \leq \infty
$$

and

$$
\left\|h^{*} f\right\|_{L^{p}\left(\mathbb{R}^{+}\right)} \leq p\|f\|_{L^{p}\left(\mathbb{R}^{+}\right)}, \quad 1 \leq p<\infty .
$$

In addition, Hardy proved that the constants $p$ and $\frac{p}{p-1}$ above are the best possible; hence,

$$
\|h\|_{L^{p}\left(\mathbb{R}^{+}\right) \rightarrow L^{p}\left(\mathbb{R}^{+}\right)}=\frac{p}{p-1}, \quad 1<p \leq \infty
$$

Copyright 2016 by the Tusi Mathematical Research Group.

Received Aug. 4, 2015; Accepted Dec. 28, 2015.

${ }^{*}$ Corresponding author.

2010 Mathematics Subject Classification. Primary 42B35; Secondary 26D10, 46E30, 26 D15.

Keywords. Hardy operator, the conjugate Hardy operator, central Morrey space, weak central Morrey space, duality. 


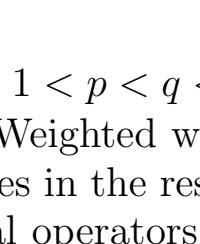

ANALYSIS
Ann. Funct. Anal. 7 (2016), no. 3, 421-433

http://dx.doi.org/10.1215/20088752-3605447

ISSN: 2008-8752 (electronic)

http://projecteuclid.org/afa

\title{
SHARP WEAK ESTIMATES FOR HARDY-TYPE OPERATORS
}

\author{
GUILIAN GAO, XIAOMIN HU, ${ }^{*}$ and CHUNJIE ZHANG \\ Communicated by J. Soria
}

\begin{abstract}
In this article, we prove the weak bound for an $n$-dimensional Hardy operator on a central Morrey space. Meanwhile, we obtain the precise operator norm, and we give the weak bounds for the conjugate Hardy operator on Lebesgue space with power weights. The corresponding operator norms are also computed. As an application, we obtain an estimate for the gamma function.
\end{abstract}

\section{INTRODUCTION}

Let $h$ and $h^{*}$ be the Hardy averaging operator and its conjugate Hardy operator,

$$
h f(x)=\frac{1}{x} \int_{0}^{x} f(t) d t, \quad h^{*} f(x)=\int_{x}^{\infty} \frac{f(t)}{t} d t, \quad x>0,
$$

respectively. The classical Hardy inequalities are

$$
\|h f\|_{L^{p}\left(\mathbb{R}^{+}\right)} \leq \frac{p}{p-1}\|f\|_{L^{p}\left(\mathbb{R}^{+}\right)}, \quad 1<p \leq \infty
$$

and

$$
\left\|h^{*} f\right\|_{L^{p}\left(\mathbb{R}^{+}\right)} \leq p\|f\|_{L^{p}\left(\mathbb{R}^{+}\right)}, \quad 1 \leq p<\infty .
$$

In addition, Hardy proved that the constants $p$ and $\frac{p}{p-1}$ above are the best possible; hence,

$$
\|h\|_{L^{p}\left(\mathbb{R}^{+}\right) \rightarrow L^{p}\left(\mathbb{R}^{+}\right)}=\frac{p}{p-1}, \quad 1<p \leq \infty
$$

Copyright 2016 by the Tusi Mathematical Research Group.

Received Aug. 4, 2015; Accepted Dec. 28, 2015.

${ }^{*}$ Corresponding author.

2010 Mathematics Subject Classification. Primary 42B35; Secondary 26D10, 46E30, 26 D15.

Keywords. Hardy operator, the conjugate Hardy operator, central Morrey space, weak central Morrey space, duality. 


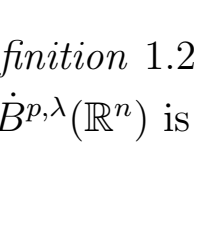

ANALYSIS
Ann. Funct. Anal. 7 (2016), no. 3, 421-433

http://dx.doi.org/10.1215/20088752-3605447

ISSN: 2008-8752 (electronic)

http://projecteuclid.org/afa

\title{
SHARP WEAK ESTIMATES FOR HARDY-TYPE OPERATORS
}

\author{
GUILIAN GAO, XIAOMIN HU, ${ }^{*}$ and CHUNJIE ZHANG \\ Communicated by J. Soria
}

\begin{abstract}
In this article, we prove the weak bound for an $n$-dimensional Hardy operator on a central Morrey space. Meanwhile, we obtain the precise operator norm, and we give the weak bounds for the conjugate Hardy operator on Lebesgue space with power weights. The corresponding operator norms are also computed. As an application, we obtain an estimate for the gamma function.
\end{abstract}

\section{INTRODUCTION}

Let $h$ and $h^{*}$ be the Hardy averaging operator and its conjugate Hardy operator,

$$
h f(x)=\frac{1}{x} \int_{0}^{x} f(t) d t, \quad h^{*} f(x)=\int_{x}^{\infty} \frac{f(t)}{t} d t, \quad x>0,
$$

respectively. The classical Hardy inequalities are

$$
\|h f\|_{L^{p}\left(\mathbb{R}^{+}\right)} \leq \frac{p}{p-1}\|f\|_{L^{p}\left(\mathbb{R}^{+}\right)}, \quad 1<p \leq \infty
$$

and

$$
\left\|h^{*} f\right\|_{L^{p}\left(\mathbb{R}^{+}\right)} \leq p\|f\|_{L^{p}\left(\mathbb{R}^{+}\right)}, \quad 1 \leq p<\infty .
$$

In addition, Hardy proved that the constants $p$ and $\frac{p}{p-1}$ above are the best possible; hence,

$$
\|h\|_{L^{p}\left(\mathbb{R}^{+}\right) \rightarrow L^{p}\left(\mathbb{R}^{+}\right)}=\frac{p}{p-1}, \quad 1<p \leq \infty
$$

Copyright 2016 by the Tusi Mathematical Research Group.

Received Aug. 4, 2015; Accepted Dec. 28, 2015.

${ }^{*}$ Corresponding author.

2010 Mathematics Subject Classification. Primary 42B35; Secondary 26D10, 46E30, 26 D15.

Keywords. Hardy operator, the conjugate Hardy operator, central Morrey space, weak central Morrey space, duality. 


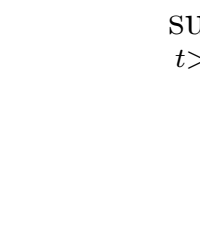

ANALYSIS
Ann. Funct. Anal. 7 (2016), no. 3, 421-433

http://dx.doi.org/10.1215/20088752-3605447

ISSN: 2008-8752 (electronic)

http://projecteuclid.org/afa

\title{
SHARP WEAK ESTIMATES FOR HARDY-TYPE OPERATORS
}

\author{
GUILIAN GAO, XIAOMIN HU, ${ }^{*}$ and CHUNJIE ZHANG \\ Communicated by J. Soria
}

\begin{abstract}
In this article, we prove the weak bound for an $n$-dimensional Hardy operator on a central Morrey space. Meanwhile, we obtain the precise operator norm, and we give the weak bounds for the conjugate Hardy operator on Lebesgue space with power weights. The corresponding operator norms are also computed. As an application, we obtain an estimate for the gamma function.
\end{abstract}

\section{INTRODUCTION}

Let $h$ and $h^{*}$ be the Hardy averaging operator and its conjugate Hardy operator,

$$
h f(x)=\frac{1}{x} \int_{0}^{x} f(t) d t, \quad h^{*} f(x)=\int_{x}^{\infty} \frac{f(t)}{t} d t, \quad x>0,
$$

respectively. The classical Hardy inequalities are

$$
\|h f\|_{L^{p}\left(\mathbb{R}^{+}\right)} \leq \frac{p}{p-1}\|f\|_{L^{p}\left(\mathbb{R}^{+}\right)}, \quad 1<p \leq \infty
$$

and

$$
\left\|h^{*} f\right\|_{L^{p}\left(\mathbb{R}^{+}\right)} \leq p\|f\|_{L^{p}\left(\mathbb{R}^{+}\right)}, \quad 1 \leq p<\infty .
$$

In addition, Hardy proved that the constants $p$ and $\frac{p}{p-1}$ above are the best possible; hence,

$$
\|h\|_{L^{p}\left(\mathbb{R}^{+}\right) \rightarrow L^{p}\left(\mathbb{R}^{+}\right)}=\frac{p}{p-1}, \quad 1<p \leq \infty
$$

Copyright 2016 by the Tusi Mathematical Research Group.

Received Aug. 4, 2015; Accepted Dec. 28, 2015.

${ }^{*}$ Corresponding author.

2010 Mathematics Subject Classification. Primary 42B35; Secondary 26D10, 46E30, 26 D15.

Keywords. Hardy operator, the conjugate Hardy operator, central Morrey space, weak central Morrey space, duality. 


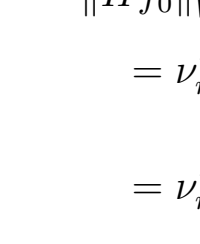

ANALYSIS
Ann. Funct. Anal. 7 (2016), no. 3, 421-433

http://dx.doi.org/10.1215/20088752-3605447

ISSN: 2008-8752 (electronic)

http://projecteuclid.org/afa

\title{
SHARP WEAK ESTIMATES FOR HARDY-TYPE OPERATORS
}

\author{
GUILIAN GAO, XIAOMIN HU, ${ }^{*}$ and CHUNJIE ZHANG \\ Communicated by J. Soria
}

\begin{abstract}
In this article, we prove the weak bound for an $n$-dimensional Hardy operator on a central Morrey space. Meanwhile, we obtain the precise operator norm, and we give the weak bounds for the conjugate Hardy operator on Lebesgue space with power weights. The corresponding operator norms are also computed. As an application, we obtain an estimate for the gamma function.
\end{abstract}

\section{INTRODUCTION}

Let $h$ and $h^{*}$ be the Hardy averaging operator and its conjugate Hardy operator,

$$
h f(x)=\frac{1}{x} \int_{0}^{x} f(t) d t, \quad h^{*} f(x)=\int_{x}^{\infty} \frac{f(t)}{t} d t, \quad x>0,
$$

respectively. The classical Hardy inequalities are

$$
\|h f\|_{L^{p}\left(\mathbb{R}^{+}\right)} \leq \frac{p}{p-1}\|f\|_{L^{p}\left(\mathbb{R}^{+}\right)}, \quad 1<p \leq \infty
$$

and

$$
\left\|h^{*} f\right\|_{L^{p}\left(\mathbb{R}^{+}\right)} \leq p\|f\|_{L^{p}\left(\mathbb{R}^{+}\right)}, \quad 1 \leq p<\infty .
$$

In addition, Hardy proved that the constants $p$ and $\frac{p}{p-1}$ above are the best possible; hence,

$$
\|h\|_{L^{p}\left(\mathbb{R}^{+}\right) \rightarrow L^{p}\left(\mathbb{R}^{+}\right)}=\frac{p}{p-1}, \quad 1<p \leq \infty
$$

Copyright 2016 by the Tusi Mathematical Research Group.

Received Aug. 4, 2015; Accepted Dec. 28, 2015.

${ }^{*}$ Corresponding author.

2010 Mathematics Subject Classification. Primary 42B35; Secondary 26D10, 46E30, 26 D15.

Keywords. Hardy operator, the conjugate Hardy operator, central Morrey space, weak central Morrey space, duality. 


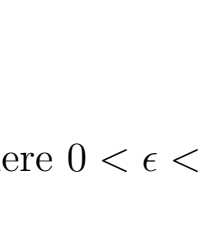

ANALYSIS
Ann. Funct. Anal. 7 (2016), no. 3, 421-433

http://dx.doi.org/10.1215/20088752-3605447

ISSN: 2008-8752 (electronic)

http://projecteuclid.org/afa

\title{
SHARP WEAK ESTIMATES FOR HARDY-TYPE OPERATORS
}

\author{
GUILIAN GAO, XIAOMIN HU, ${ }^{*}$ and CHUNJIE ZHANG \\ Communicated by J. Soria
}

\begin{abstract}
In this article, we prove the weak bound for an $n$-dimensional Hardy operator on a central Morrey space. Meanwhile, we obtain the precise operator norm, and we give the weak bounds for the conjugate Hardy operator on Lebesgue space with power weights. The corresponding operator norms are also computed. As an application, we obtain an estimate for the gamma function.
\end{abstract}

\section{INTRODUCTION}

Let $h$ and $h^{*}$ be the Hardy averaging operator and its conjugate Hardy operator,

$$
h f(x)=\frac{1}{x} \int_{0}^{x} f(t) d t, \quad h^{*} f(x)=\int_{x}^{\infty} \frac{f(t)}{t} d t, \quad x>0,
$$

respectively. The classical Hardy inequalities are

$$
\|h f\|_{L^{p}\left(\mathbb{R}^{+}\right)} \leq \frac{p}{p-1}\|f\|_{L^{p}\left(\mathbb{R}^{+}\right)}, \quad 1<p \leq \infty
$$

and

$$
\left\|h^{*} f\right\|_{L^{p}\left(\mathbb{R}^{+}\right)} \leq p\|f\|_{L^{p}\left(\mathbb{R}^{+}\right)}, \quad 1 \leq p<\infty .
$$

In addition, Hardy proved that the constants $p$ and $\frac{p}{p-1}$ above are the best possible; hence,

$$
\|h\|_{L^{p}\left(\mathbb{R}^{+}\right) \rightarrow L^{p}\left(\mathbb{R}^{+}\right)}=\frac{p}{p-1}, \quad 1<p \leq \infty
$$

Copyright 2016 by the Tusi Mathematical Research Group.

Received Aug. 4, 2015; Accepted Dec. 28, 2015.

${ }^{*}$ Corresponding author.

2010 Mathematics Subject Classification. Primary 42B35; Secondary 26D10, 46E30, 26 D15.

Keywords. Hardy operator, the conjugate Hardy operator, central Morrey space, weak central Morrey space, duality. 


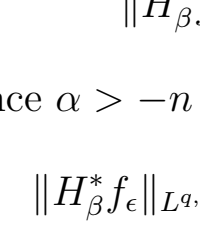

ANALYSIS
Ann. Funct. Anal. 7 (2016), no. 3, 421-433

http://dx.doi.org/10.1215/20088752-3605447

ISSN: 2008-8752 (electronic)

http://projecteuclid.org/afa

\title{
SHARP WEAK ESTIMATES FOR HARDY-TYPE OPERATORS
}

\author{
GUILIAN GAO, XIAOMIN HU, ${ }^{*}$ and CHUNJIE ZHANG \\ Communicated by J. Soria
}

\begin{abstract}
In this article, we prove the weak bound for an $n$-dimensional Hardy operator on a central Morrey space. Meanwhile, we obtain the precise operator norm, and we give the weak bounds for the conjugate Hardy operator on Lebesgue space with power weights. The corresponding operator norms are also computed. As an application, we obtain an estimate for the gamma function.
\end{abstract}

\section{INTRODUCTION}

Let $h$ and $h^{*}$ be the Hardy averaging operator and its conjugate Hardy operator,

$$
h f(x)=\frac{1}{x} \int_{0}^{x} f(t) d t, \quad h^{*} f(x)=\int_{x}^{\infty} \frac{f(t)}{t} d t, \quad x>0,
$$

respectively. The classical Hardy inequalities are

$$
\|h f\|_{L^{p}\left(\mathbb{R}^{+}\right)} \leq \frac{p}{p-1}\|f\|_{L^{p}\left(\mathbb{R}^{+}\right)}, \quad 1<p \leq \infty
$$

and

$$
\left\|h^{*} f\right\|_{L^{p}\left(\mathbb{R}^{+}\right)} \leq p\|f\|_{L^{p}\left(\mathbb{R}^{+}\right)}, \quad 1 \leq p<\infty .
$$

In addition, Hardy proved that the constants $p$ and $\frac{p}{p-1}$ above are the best possible; hence,

$$
\|h\|_{L^{p}\left(\mathbb{R}^{+}\right) \rightarrow L^{p}\left(\mathbb{R}^{+}\right)}=\frac{p}{p-1}, \quad 1<p \leq \infty
$$

Copyright 2016 by the Tusi Mathematical Research Group.

Received Aug. 4, 2015; Accepted Dec. 28, 2015.

${ }^{*}$ Corresponding author.

2010 Mathematics Subject Classification. Primary 42B35; Secondary 26D10, 46E30, 26 D15.

Keywords. Hardy operator, the conjugate Hardy operator, central Morrey space, weak central Morrey space, duality. 


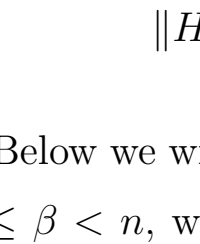

ANALYSIS
Ann. Funct. Anal. 7 (2016), no. 3, 421-433

http://dx.doi.org/10.1215/20088752-3605447

ISSN: 2008-8752 (electronic)

http://projecteuclid.org/afa

\title{
SHARP WEAK ESTIMATES FOR HARDY-TYPE OPERATORS
}

\author{
GUILIAN GAO, XIAOMIN HU, ${ }^{*}$ and CHUNJIE ZHANG \\ Communicated by J. Soria
}

\begin{abstract}
In this article, we prove the weak bound for an $n$-dimensional Hardy operator on a central Morrey space. Meanwhile, we obtain the precise operator norm, and we give the weak bounds for the conjugate Hardy operator on Lebesgue space with power weights. The corresponding operator norms are also computed. As an application, we obtain an estimate for the gamma function.
\end{abstract}

\section{INTRODUCTION}

Let $h$ and $h^{*}$ be the Hardy averaging operator and its conjugate Hardy operator,

$$
h f(x)=\frac{1}{x} \int_{0}^{x} f(t) d t, \quad h^{*} f(x)=\int_{x}^{\infty} \frac{f(t)}{t} d t, \quad x>0,
$$

respectively. The classical Hardy inequalities are

$$
\|h f\|_{L^{p}\left(\mathbb{R}^{+}\right)} \leq \frac{p}{p-1}\|f\|_{L^{p}\left(\mathbb{R}^{+}\right)}, \quad 1<p \leq \infty
$$

and

$$
\left\|h^{*} f\right\|_{L^{p}\left(\mathbb{R}^{+}\right)} \leq p\|f\|_{L^{p}\left(\mathbb{R}^{+}\right)}, \quad 1 \leq p<\infty .
$$

In addition, Hardy proved that the constants $p$ and $\frac{p}{p-1}$ above are the best possible; hence,

$$
\|h\|_{L^{p}\left(\mathbb{R}^{+}\right) \rightarrow L^{p}\left(\mathbb{R}^{+}\right)}=\frac{p}{p-1}, \quad 1<p \leq \infty
$$

Copyright 2016 by the Tusi Mathematical Research Group.

Received Aug. 4, 2015; Accepted Dec. 28, 2015.

${ }^{*}$ Corresponding author.

2010 Mathematics Subject Classification. Primary 42B35; Secondary 26D10, 46E30, 26 D15.

Keywords. Hardy operator, the conjugate Hardy operator, central Morrey space, weak central Morrey space, duality. 


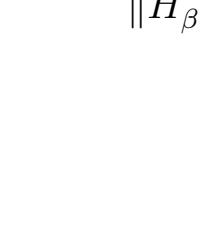

ANALYSIS
Ann. Funct. Anal. 7 (2016), no. 3, 421-433

http://dx.doi.org/10.1215/20088752-3605447

ISSN: 2008-8752 (electronic)

http://projecteuclid.org/afa

\title{
SHARP WEAK ESTIMATES FOR HARDY-TYPE OPERATORS
}

\author{
GUILIAN GAO, XIAOMIN HU, ${ }^{*}$ and CHUNJIE ZHANG \\ Communicated by J. Soria
}

\begin{abstract}
In this article, we prove the weak bound for an $n$-dimensional Hardy operator on a central Morrey space. Meanwhile, we obtain the precise operator norm, and we give the weak bounds for the conjugate Hardy operator on Lebesgue space with power weights. The corresponding operator norms are also computed. As an application, we obtain an estimate for the gamma function.
\end{abstract}

\section{INTRODUCTION}

Let $h$ and $h^{*}$ be the Hardy averaging operator and its conjugate Hardy operator,

$$
h f(x)=\frac{1}{x} \int_{0}^{x} f(t) d t, \quad h^{*} f(x)=\int_{x}^{\infty} \frac{f(t)}{t} d t, \quad x>0,
$$

respectively. The classical Hardy inequalities are

$$
\|h f\|_{L^{p}\left(\mathbb{R}^{+}\right)} \leq \frac{p}{p-1}\|f\|_{L^{p}\left(\mathbb{R}^{+}\right)}, \quad 1<p \leq \infty
$$

and

$$
\left\|h^{*} f\right\|_{L^{p}\left(\mathbb{R}^{+}\right)} \leq p\|f\|_{L^{p}\left(\mathbb{R}^{+}\right)}, \quad 1 \leq p<\infty .
$$

In addition, Hardy proved that the constants $p$ and $\frac{p}{p-1}$ above are the best possible; hence,

$$
\|h\|_{L^{p}\left(\mathbb{R}^{+}\right) \rightarrow L^{p}\left(\mathbb{R}^{+}\right)}=\frac{p}{p-1}, \quad 1<p \leq \infty
$$

Copyright 2016 by the Tusi Mathematical Research Group.

Received Aug. 4, 2015; Accepted Dec. 28, 2015.

${ }^{*}$ Corresponding author.

2010 Mathematics Subject Classification. Primary 42B35; Secondary 26D10, 46E30, 26 D15.

Keywords. Hardy operator, the conjugate Hardy operator, central Morrey space, weak central Morrey space, duality. 


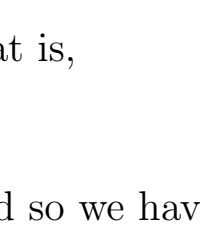

ANALYSIS
Ann. Funct. Anal. 7 (2016), no. 3, 421-433

http://dx.doi.org/10.1215/20088752-3605447

ISSN: 2008-8752 (electronic)

http://projecteuclid.org/afa

\title{
SHARP WEAK ESTIMATES FOR HARDY-TYPE OPERATORS
}

\author{
GUILIAN GAO, XIAOMIN HU, ${ }^{*}$ and CHUNJIE ZHANG \\ Communicated by J. Soria
}

\begin{abstract}
In this article, we prove the weak bound for an $n$-dimensional Hardy operator on a central Morrey space. Meanwhile, we obtain the precise operator norm, and we give the weak bounds for the conjugate Hardy operator on Lebesgue space with power weights. The corresponding operator norms are also computed. As an application, we obtain an estimate for the gamma function.
\end{abstract}

\section{INTRODUCTION}

Let $h$ and $h^{*}$ be the Hardy averaging operator and its conjugate Hardy operator,

$$
h f(x)=\frac{1}{x} \int_{0}^{x} f(t) d t, \quad h^{*} f(x)=\int_{x}^{\infty} \frac{f(t)}{t} d t, \quad x>0,
$$

respectively. The classical Hardy inequalities are

$$
\|h f\|_{L^{p}\left(\mathbb{R}^{+}\right)} \leq \frac{p}{p-1}\|f\|_{L^{p}\left(\mathbb{R}^{+}\right)}, \quad 1<p \leq \infty
$$

and

$$
\left\|h^{*} f\right\|_{L^{p}\left(\mathbb{R}^{+}\right)} \leq p\|f\|_{L^{p}\left(\mathbb{R}^{+}\right)}, \quad 1 \leq p<\infty .
$$

In addition, Hardy proved that the constants $p$ and $\frac{p}{p-1}$ above are the best possible; hence,

$$
\|h\|_{L^{p}\left(\mathbb{R}^{+}\right) \rightarrow L^{p}\left(\mathbb{R}^{+}\right)}=\frac{p}{p-1}, \quad 1<p \leq \infty
$$

Copyright 2016 by the Tusi Mathematical Research Group.

Received Aug. 4, 2015; Accepted Dec. 28, 2015.

${ }^{*}$ Corresponding author.

2010 Mathematics Subject Classification. Primary 42B35; Secondary 26D10, 46E30, 26 D15.

Keywords. Hardy operator, the conjugate Hardy operator, central Morrey space, weak central Morrey space, duality. 


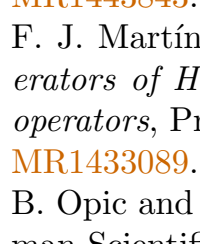

ANALYSIS
Ann. Funct. Anal. 7 (2016), no. 3, 421-433

http://dx.doi.org/10.1215/20088752-3605447

ISSN: 2008-8752 (electronic)

http://projecteuclid.org/afa

\title{
SHARP WEAK ESTIMATES FOR HARDY-TYPE OPERATORS
}

\author{
GUILIAN GAO, XIAOMIN HU, ${ }^{*}$ and CHUNJIE ZHANG \\ Communicated by J. Soria
}

\begin{abstract}
In this article, we prove the weak bound for an $n$-dimensional Hardy operator on a central Morrey space. Meanwhile, we obtain the precise operator norm, and we give the weak bounds for the conjugate Hardy operator on Lebesgue space with power weights. The corresponding operator norms are also computed. As an application, we obtain an estimate for the gamma function.
\end{abstract}

\section{INTRODUCTION}

Let $h$ and $h^{*}$ be the Hardy averaging operator and its conjugate Hardy operator,

$$
h f(x)=\frac{1}{x} \int_{0}^{x} f(t) d t, \quad h^{*} f(x)=\int_{x}^{\infty} \frac{f(t)}{t} d t, \quad x>0,
$$

respectively. The classical Hardy inequalities are

$$
\|h f\|_{L^{p}\left(\mathbb{R}^{+}\right)} \leq \frac{p}{p-1}\|f\|_{L^{p}\left(\mathbb{R}^{+}\right)}, \quad 1<p \leq \infty
$$

and

$$
\left\|h^{*} f\right\|_{L^{p}\left(\mathbb{R}^{+}\right)} \leq p\|f\|_{L^{p}\left(\mathbb{R}^{+}\right)}, \quad 1 \leq p<\infty .
$$

In addition, Hardy proved that the constants $p$ and $\frac{p}{p-1}$ above are the best possible; hence,

$$
\|h\|_{L^{p}\left(\mathbb{R}^{+}\right) \rightarrow L^{p}\left(\mathbb{R}^{+}\right)}=\frac{p}{p-1}, \quad 1<p \leq \infty
$$

Copyright 2016 by the Tusi Mathematical Research Group.

Received Aug. 4, 2015; Accepted Dec. 28, 2015.

${ }^{*}$ Corresponding author.

2010 Mathematics Subject Classification. Primary 42B35; Secondary 26D10, 46E30, 26 D15.

Keywords. Hardy operator, the conjugate Hardy operator, central Morrey space, weak central Morrey space, duality. 